\title{
Predicting Unconventional High-Temperature Superconductors in Trigonal Bipyramidal Coordinations
}

\author{
Jiangping $\mathrm{Hu},{ }^{1,2,3, *}$ Congcong Le, ${ }^{1}$ and Xianxin $\mathrm{Wu}^{1}$ \\ ${ }^{1}$ Beijing National Laboratory for Condensed Matter Physics, and Institute of Physics, \\ Chinese Academy of Sciences, Beijing 100190, China \\ ${ }^{2}$ Department of Physics, Purdue University, West Lafayette, Indiana 47907, USA \\ ${ }^{3}$ Collaborative Innovation Center of Quantum Matter, Beijing 100871, China
}

(Received 24 June 2015; revised manuscript received 18 August 2015; published 23 October 2015)

\begin{abstract}
Cuprates and iron-based superconductors are two classes of unconventional high- $T_{c}$ superconductors based on $3 d$ transition elements. Recently, two principles, the correspondence principle and the magnetic selective pairing rule, have emerged to unify their high- $T_{c}$ superconducting mechanisms. These principles strongly regulate electronic structures that can host high- $T_{c}$ superconductivity. Guided by these principles, here, we propose high- $T_{c}$ superconducting candidates that are formed by cation-anion trigonal bipyramidal complexes with a $d^{7}$ filling configuration on the cation ions. Their superconducting states are expected to be dominated by the $d_{x y} \pm i d_{x^{2}-y^{2}}$ pairing symmetry.
\end{abstract}

DOI: 10.1103/PhysRevX.5.041012

Almost three decades ago, cuprates [1], the $\mathrm{Cu}$-based high$T_{c}$ superconductors, were discovered. Since then, understanding the superconducting mechanism behind unconventional high-temperature superconductors has become a great challenge in condensed-matter physics. In the past six years, new light has been shone onto this decades-old problem due to the discovery of iron-based high- $T_{c}$ superconductors [2]. The two high-temperature superconductors share many common electronic properties [3]. In principle, comparing these two classes of materials, we may determine the key ingredients that are essential to the high- $T_{c}$ superconducting mechanism. However, even if we have identified them, without a realistic prediction of new high- $T_{c}$ superconductors, reaching a final consensus will be extremely difficult.

Most recently, one of us emphasized and proposed two basic principles to unify the understanding for both high- $T_{c}$ superconductors [4]: (1) The correspondence principle, which was first specified in Ref. [5] by $\mathrm{Hu}$ and Ding and was generalized to include other orders later in Ref. [6] by Davis and Lee, notes that the short-range magnetic exchange interactions and the Fermi surfaces act collaboratively to achieve high- $T_{c}$ superconductivity and determine pairing symmetries. (2) The selective magnetic pairing rule notes that the superconductivity is only induced by the magnetic exchange couplings from the

\footnotetext{
*jphu@iphy.ac.cn
}

Published by the American Physical Society under the terms of the Creative Commons Attribution 3.0 License. Further distribution of this work must maintain attribution to the author(s) and the published article's title, journal citation, and DOI.
Subject Areas: Condensed Matter Physics,

Materials Science,

Strongly Correlated Materials

superexchange mechanism through cation-anion-cation chemical bonds but not those from direct exchange couplings resulted from the direct cation's $d$ - $d$ chemical bonds. These two principles provide an unified explanation why the $d$-wave pairing symmetry and the $s$-wave pairing symmetry are robust, respectively, in cuprates and ironbased superconductors [4]. In the meantime, the above two principles can serve as direct guiding rules to search for high- $T_{c}$ superconductors. The two principles provide many constraints on electronic structures that can host high- $T_{c}$ superconductivity. The detailed summary of these constraints and their microscopic origins were discussed in Ref. [4]. Essentially, the two principles suggest that the quasi-two dimensional electronic environment that hosts high $T_{c}$ superconductivity must be dominated by the $d$ orbitals with the strongest in-plane $d$ - $p$ hybridization near Fermi energy.

Here, guided by these principles, combining with crystal field theory and first-principles calculations, we predict a new electronic structure that can host high- $T_{c}$ superconductivity with $d \pm i d$ pairing symmetry.

We start to search for possible high- $T_{c}$ candidates by analyzing the basic building blocks, namely, the cationanion complexes. Taking both cuprates and iron-based superconductors as examples, we check how the principles are satisfied in these two superconductors. As shown in Fig. 1(a), the $\mathrm{Cu}$ atoms in cuprates are in an octahedral complex. In this complex, the five $d$ orbitals split into two groups by crystal fields $t_{2 g}$ and $e_{g}$. The two orbitals in the $e_{g}$ group $d_{z^{2}}$ and $d_{x^{2}-y^{2}}$, because of their strong couplings to the $p$ orbitals of the surrounding oxygen atoms, have higher energies. In the $\mathrm{Cu}-\mathrm{O}$ plane of cuprates formed by the 


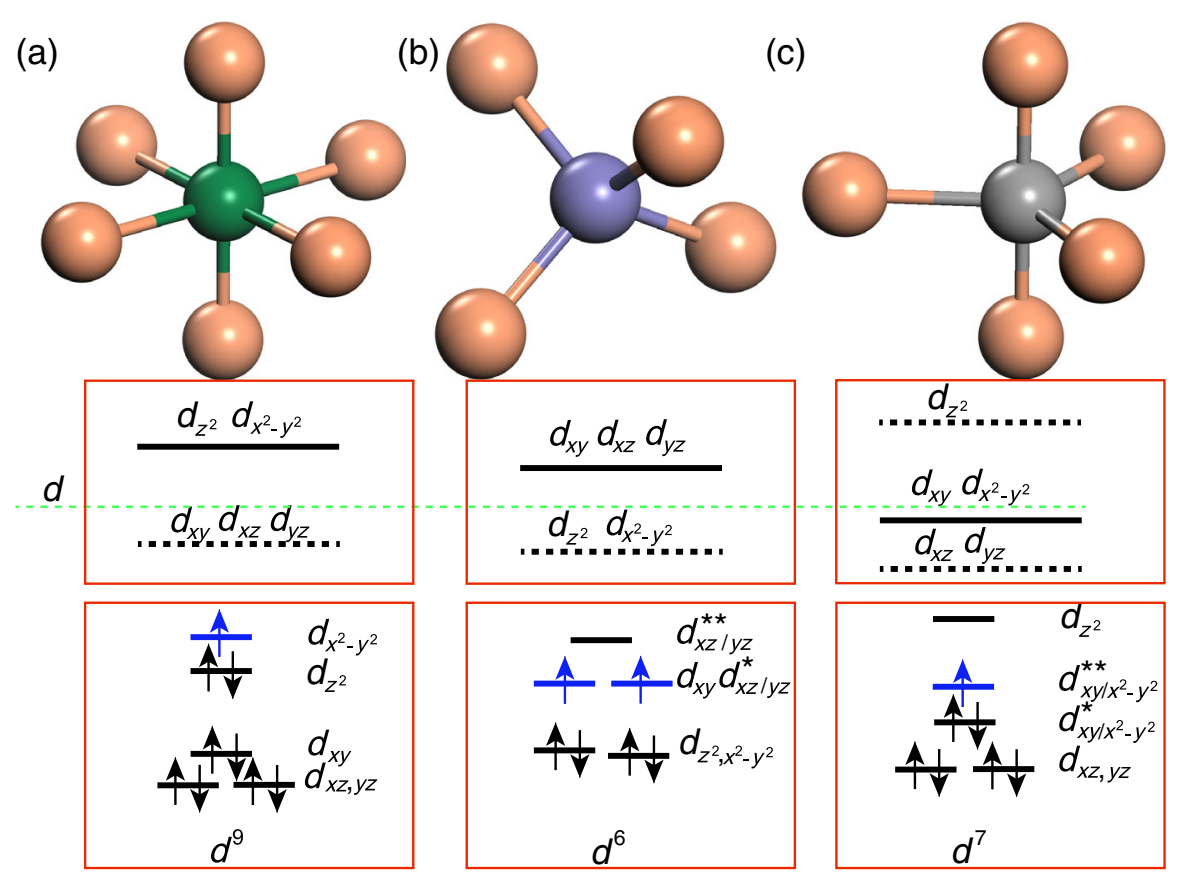

FIG. 1. Structural units, crystal field splitting in one unit complex and energy splitting in a two-dimensional lattice structure created by the corresponding complexes. (a) Octahedral complex (cuprates, $\mathrm{CuO}_{6}$, the $\mathrm{Cu}-\mathrm{O}$ plane). (b) Tetrahedral complex (iron-based superconductors, $\mathrm{FeAs}_{4} / \mathrm{Se}_{4}$, the FeAs/Se layer). (c) Trigonal bipyramidal complex (Ni/CoO 3 , the Ni/Co-O triangular layer). The $d$ orbitals with the blue color are active for superconductivity.

octahedral complexes, however, only the $d_{x^{2}-y^{2}}$ orbitals have strong in-plane couplings to the $p$ orbitals. Therefore, following the above rule, only the electronic band attributed to the $d_{x^{2}-y^{2}}$ orbitals can support high- $T_{c}$ superconductivity. The $d_{z^{2}}$ energy level is lowered due to the Jahn-Teller effect, and the $d_{x^{2}-y^{2}}$ orbital is the single orbital at the highest energy, as shown in Fig. 1(a). Thus, it is easy to see that in this case, an electronic band structure for high- $T_{c}$ superconductors can only be achieved under the $3 d^{9}\left(\mathrm{Cu}^{2+}\right)$ configuration. In iron-based superconductors, the $\mathrm{Fe}$ atoms are in a tetrahedral complex. Compared with the octahedral environment, the energy levels of the $t_{2 g}$ and $e_{g}$ orbitals in the tetrahedral complex reverse. The $t_{2 g}$ orbitals have higher energy because of their strong couplings to the $\mathrm{As} / \mathrm{Se}$ anions. If we further consider two molecular orbitals formed by $d_{x z}$ and $d_{y z}$, one molecular orbital is strongly coupled to the $e_{g}$ orbitals and gains energy. This orbital, labeled as $d_{x z / y z}^{* *}$ in Fig. 1(b), is thus heavily dressed by $d_{x^{2}-y^{2}}$. The $d_{x y}$ orbital and the other orbital formed by $d_{x z}$ and $d_{y z}$ are the only two orbitals that can provide electronic environment to host possible high- $T_{c}$ superconductivity. Thus, as shown in Fig. 1(b), the $3 d^{6}\left(\mathrm{Fe}^{2+}\right)$ configuration is the filling level to make these pure $t_{2 g}$ orbitals dominate electronic band structures close to Fermi energy. The high- $T_{c}$ superconductivity is thus only achieved under the $3 d^{6}$ configuration. From these understandings, we can see that the two principles fix the $d$-orbital filling configuration if a structure formed by a given cation-anion complex is a high- $T_{c}$ superconductivity candidate. This result partially explains why high- $T_{c}$ superconductivity appears to be such a rare phenomenon.

If we compare all cation-anion complexes, the trigonal bipyramidal complex has slightly lower symmetry than the octahedral or tetrahedral complexes and is characterized by five coordinations, a number just between six coordinations in octahedra and four coordinations in tetrahedra. Materials with layered structures have also been formed by trigonal bipyramidal complexes, such as $\mathrm{YMnO}_{3}[7,8]$, in which $\mathrm{Mn}$ atoms in a Mn-O hexagonal lattice form a triangular lattice through corner-shared $\mathrm{MnO}_{5}$ complexes, as shown in Fig. 2. The $d$ orbitals in the trigonal bipyramidal complex are split into three groups, as shown in Fig. 1(c). The $d_{z^{2}}$ orbital has the highest energy due to its strong couplings to apical anions. The degenerate $d_{x^{2}-y^{2}}$ and $d_{x y}$ orbitals are strongly coupled to the in-plane anions. The degenerate $d_{x z}$ and $d_{y z}$ orbitals have the lowest energy and are only weakly coupled to anions. Thus, one can guess that a $3 d^{6}$ or $3 d^{7}$ configuration may result in a possible band structure in which the $d_{x^{2}-y^{2}}$ and $d_{x y}$ orbitals dominate near Fermi surfaces. If we further consider two molecular orbitals formed by the $d_{x^{2}-y^{2}}$ and $d_{x y}$ orbitals, one of them can strongly couple to the $d_{z^{2}}$ in a three-corner-shared trigonal structure, which will be shown later. As the $d_{z^{2}}$ orbital has higher energy, the coupling lowers the energy level of this molecular orbital. Therefore, to form a band structure that is dominated by the pure $d_{x^{2}-y^{2}}$ and $d_{x y}$ orbitals near Fermi energy, the $3 d^{7}$ filling configuration is expected, as shown in Fig. 1(c). 


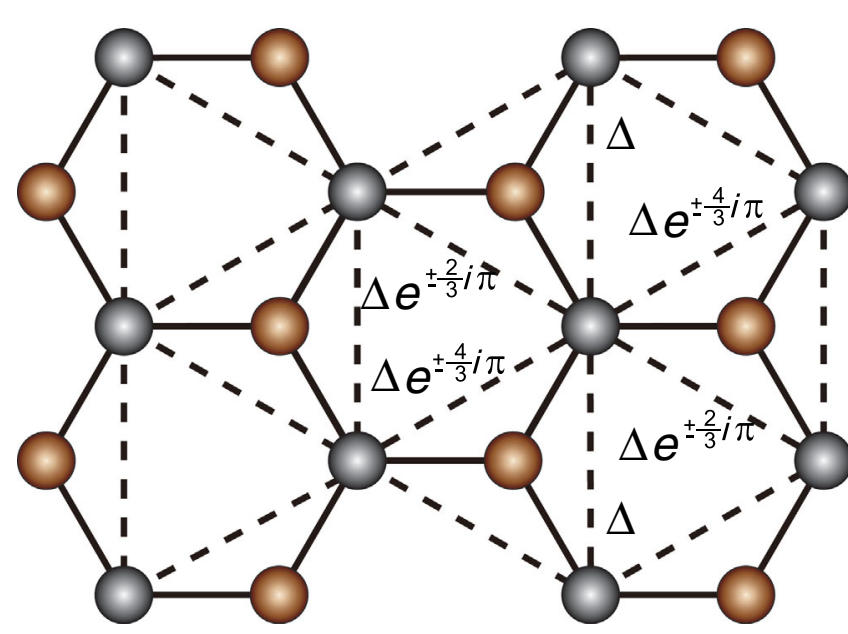

FIG. 2. The two-dimensional hexagonal lattice formed by the corner-shared trigonal bipyramidal complexes. The gray cation atoms further form a triangle lattice. The superconducting pairing configuration in a $d \pm i d$ pairing state is sketched.

Both $\mathrm{Co}^{2+}$ and $\mathrm{Ni}^{3+}$ ions have a $3 d^{7}$ filling configuration. The $\mathrm{MnO}_{3}$ layer in $\mathrm{YMnO}_{3}$ is the simplest prototype layer structure that can be formed by trigonal bipyramidal complexes without anion bonding. Here, we focus on this prototype structure and check whether a desired electronic structure for high- $T_{c}$ superconductivity exists. Figure 3(a) shows the electronic band structure of $\mathrm{YNiO}_{3}$. The electronic structure is rather quasi-two-dimensional and thus can be attributed to a single $\mathrm{NiO}_{3}$ layer. In Fig. 3(a), one band near the Fermi level, which has the largest dispersion and will be referred as the $\alpha$ band, is mainly attributed to the two $d_{x y}$ and $d_{x^{2}-y^{2}}$ orbitals. Another band, which will be referred to as the $\beta$ band, contributes a small hole pocket at the $\Gamma$ point. The $\beta$ band results from the bonding between the $d_{z^{2}}$ orbital and one $d_{x y, x^{2}-y^{2}}$ molecular orbital. Near the
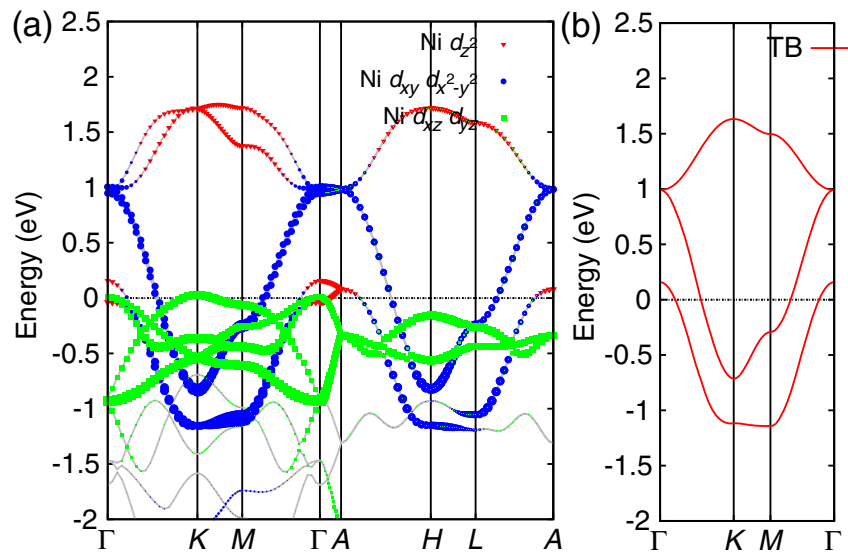

FIG. 3. (a) The band structures of $\mathrm{YNiO}_{3}$ obtained from the first-principles calculations and (b) the extracted three bands for the tight-binding model. The orbital characters of the bands in (a) are indicated by the different colors specified in the right top corner of the figure.
$\Gamma$ point, the orbital character of the $\beta$ band is mainly $d_{z^{2}}$. The other band from the antibonding between the $d_{z^{2}}$ and $d_{x y, x^{2}-y^{2}}$ orbitals, which will be referred to as the $\gamma$ band, stays at much higher energy and is mainly attributed to the $d_{z^{2}}$-orbital character. The bands from the $d_{x z}$ and $d_{y z}$ orbitals with much less dispersion are located below the Fermi level. Although it is possible that these bands may contribute small hole pockets at $K$ points, they can be assumed to be fully occupied. The $p$ orbitals of the oxygen atoms are far below the Fermi level. The large dispersion of the $d_{x y}$ and $d_{x^{2}-y^{2}}$ bands suggests a strong $d$ - $p$ hybridization. These features are consistent with the above crystal field analysis and suggest that the $3 d^{7}$ filling configuration in trigonal bipyramidal complexes is indeed a possible candidate for high-temperature superconductivity. Neglecting the interlayer coupling, the electronic structure can be well described by a three-band tightbinding (TB) model, including $d_{x y}, d_{x^{2}-y^{2}}$, and $d_{z^{2}}$ orbitals. Figure 3(b) shows that the band structure obtained from the TB model well captures the first-principles calculation results in Fig. 3(a). The corresponding hopping parameters are given in Table I. It is worth noting that the signs of intraorbital hopping parameters for the $d_{x y}$ and $d_{x^{2}-y^{2}}$ orbitals also indicate that the hopping is caused by the oxygen atoms.

Following the second principle, the $\alpha$ band from the $d_{x y}$ and $d_{x^{2}-y^{2}}$ orbitals can host high-temperature superconductivity. We can check whether this structure also satisfies the correspondence principle. Near $3 d^{7}$ filling configuration, this band is close to half filling. The $\alpha$ band can be described by a simple one-dimensional effective Hubbard or $t-J$ models in a two-dimensional triangle lattice. The dominant hopping parameter is the nearest-neighbor (NN) hopping and the short-range magnetic superexchange coupling is also the NN antiferromagnetic (AFM) exchange. In the Appendix, we also show that the AFM state has significantly lower energy than the paramagnetic state, which indicates the existence of the strong NN AFM exchange couplings.

In the parental compound $\mathrm{YNiO}_{3}$, the AFM state is favored in all generalized-gradient-approximation (GGA) $+U$ calculations by taking the values of $U$ from 0 to $8 \mathrm{eV}$. In the AFM state, the magnetic moment at $\mathrm{Ni}$ sites increases with increasing $U$ and it ranges from $0.43 \mu_{B}$ at $U=0,1.12 \mu_{B}$ at $U=4 \mathrm{eV}$, and $1.85 \mu_{B}$ at $U=8 \mathrm{eV}$.

TABLE I. The NN hopping parameters (in units of eV) along the $y$ axis in the three-orbital model. The on-site energies are $\epsilon_{1}=2.765 \mathrm{eV}$ and $\epsilon_{2}=4.186 \mathrm{eV}$ and the Fermi level is $E_{f}=3.045 \mathrm{eV}$.

\begin{tabular}{lrcr}
\hline \hline & $d_{x y}$ & $d_{x^{2}-y^{2}}$ & \multicolumn{1}{c}{$d_{z^{2}}$} \\
\hline$d_{x y}$ & 0.3147 & 0.0388 & -0.2063 \\
$d_{x^{2}-y^{2}}$ & -0.0388 & 0.1091 & 0.0678 \\
$d_{z^{2}}$ & 0.2063 & 0.0678 & -0.1639 \\
\hline \hline
\end{tabular}


With a $d^{7}$ configuration, the largest spin moment in a high spin state is $3 \mu_{B}$. Thus, as these calculations typically overestimate the magnet moments, the results suggest that $\mathrm{Ni}^{+3}$ is closer to a low spin state than to a high spin state in the reasonable parameter region. This is consistent with the crystal field theory. As shown in Fig. 1(c), in a trigonal bipyramidal complex, the crystal field splitting between $d_{z^{2}}$ and $d_{x y, x^{2}-y^{2}}$ is very large, which makes the high spin state unfavorable. A low spin state is in favor of generating superconductivity upon doping because it is relatively easy to suppress the long-range magnetic order. Furthermore, up to $U=8 \mathrm{eV}$, our calculation indicates, as shown in the Appendix, that the AFM state remains to be metallic and does not show a Mott transition. This is likely due to the presence of two closely degenerate $d$ orbitals, namely, $d_{x y}$ and $d_{x^{2}-y^{2}}$, near Fermi surfaces. The analytic understanding of this intriguing AFM metal state will be investigated elsewhere.

The superconductivity can emerge upon doping the material. In a triangle lattice, the NN AFM exchange coupling can lead to two types of pairing symmetries: the extended $s$ wave or $d \pm i d$ wave [5]. As $d_{x y}$ and $d_{x^{2}-y^{2}}$ form a two-dimensional irreducible representation of the $D_{3 h}$ point group in trigonal lattice, the two $d \pm i d$ waves are degenerate. As the pairing should be dominated on the NN bonds, for the extended $s$-wave pairing, the form factor of the gap function in the momentum space is given by $\Delta_{s} \propto \cos k_{y}+2 \cos (\sqrt{3} / 2) k_{x} \cos \frac{1}{2} k_{y}$, and similarly, for the $d \pm i d$-wave pairing, the factor is given by $\Delta_{d} \propto$ $\cos k_{y}-\cos (\sqrt{3} / 2) k_{x} \cos \frac{1}{2} k_{y} \pm i \sqrt{3} \sin (\sqrt{3} / 2) k_{x} \sin \frac{1}{2} k_{y}$. Following Ref. [5], we calculate the overlaps between the Fermi surfaces and the form factors. Figure 4 shows the overlaps for the $\alpha$ band obtained in $\mathrm{YNiO}_{3}$. It becomes obvious that the two degenerate $d \pm i d$-wave forms collaborate well with Fermi surfaces near half filling, and its overlap with the Fermi surfaces is much larger than the extended $s$-wave form. Therefore, the system is a good candidate to host a high- $T_{c}$ superconducting state with a robust $d \pm i d$-wave pairing symmetry.
The $\alpha$ band is a rather robust electronic structure as long as the two-dimensional triangle lattice is maintained. Without considering the lattice instability, we can extend the $\mathrm{YNiO}_{3}$ prototype to include many possible variations by choosing different valence anions and replacing the apical anions with different elements. In the Appendix, we provide a list of possible materials in which the $\alpha$ band stands out near the Fermi level, including $\mathrm{KNiOCl}_{2}$, $\mathrm{KNiOF}_{2}, \mathrm{BaCoOF}_{2}$, and $\mathrm{KCoF}_{3}$. In all these prototypes, the $\alpha$ is close to the half filling with a dispersion similar to the one in Fig. 3 in $\mathrm{YNiO}_{3}$. The $\beta$ and $\gamma$ bands can be tuned by changing apical anion elements. For example, in the material $\mathrm{KNiOCl}_{2}$, the $\beta$ band sinks below Fermi level and has no hole-pocket contribution at the $\Gamma$ point.

Similar to the octahedral complex, the trigonal bipyramidal complex can be flexibly crystallized into structures with multiple triangle layers in a unit cell because of the existence of the apical anions. The $\mathrm{YbFe}_{2} \mathrm{O}_{4}$ [9] structure is one such flexible structure with a double-triangle-layer structure. If we consider $\mathrm{YbNi}_{2} \mathrm{O}_{4}$ in the $3 d^{7}$ configuration, shown in the Appendix, the $\alpha$ band is very similar to the one in $\mathrm{YNiO}_{3}$. This proves again that $\alpha$ band is very robust and is strongly determined by the in-plane $d-p$ hybridization.

In cuprates, materials with multiple $\mathrm{Cu}-\mathrm{O}$ layers in a unit cell, such as $\mathrm{YBa}_{2} \mathrm{Cu}_{3} \mathrm{O}_{7-x}$ [10], have significantly higher $T_{c}$ than the single-layer materials, such as $\mathrm{La}_{2-x} \mathrm{Ba}_{x} \mathrm{CuO}_{4}$ [1]. This enhancement can be partially understood from our principles as the purification of the orbital characters near Fermi surfaces. The middle $\mathrm{Cu}-\mathrm{O}$ layer in the $\mathrm{YBa}_{2} \mathrm{Cu}_{3} \mathrm{O}_{7-x}$ unit cell is formed by plain square complexes without apical oxygens in which the energy of $d_{z^{2}}$ is further lowered to make the bands near Fermi surfaces with purer $d_{x^{2}-y^{2}}$-orbital characters. For the new structure proposed here, lowering the energy of the $d_{z}^{2}$ by partially removing apical anions will enhance the mixture of the $d_{z^{2}}$ orbital near Fermi surfaces. Therefore, in general, it is not good for achieving high $T_{c}$. However, if the apical anions are completely removed, namely, the complexes become
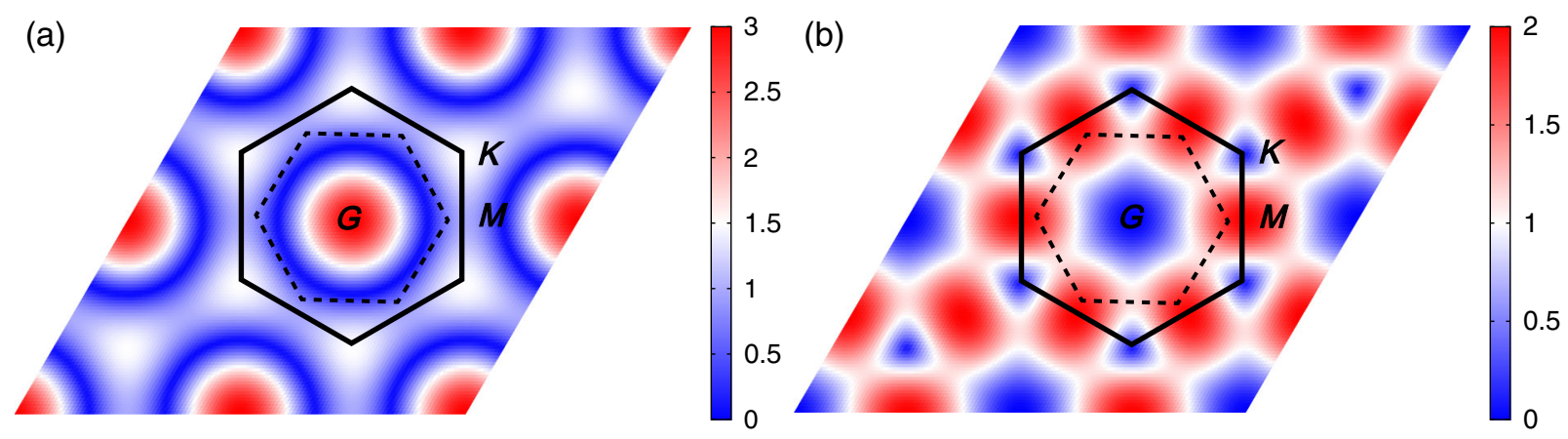

FIG. 4. The overlap between Fermi surfaces of the $\alpha$ band and gap functions. (a) The extended $s$ wave cos $k_{y}+$ $2 \cos (\sqrt{3} / 2) k_{x} \cos \frac{1}{2} k_{y}$. (b) $d \pm i d$ wave $\cos k_{y}-\cos (\sqrt{3} / 2) k_{x} \cos \frac{1}{2} k_{y} \pm i \sqrt{3} \sin (\sqrt{3} / 2) k_{x} \sin \frac{1}{2} k_{y}$. The dashed black lines represent the Fermi surfaces. The solid black lines represent the first Brillouin zone. 
plain triangular complexes, the $d_{z^{2}}$ orbital becomes the orbital with the lowest energy in crystal field splitting. In this case, it is easy to see that it may exist a $d^{8}$ filling (for example, $\mathrm{Ni}^{2+}$ ) configuration to achieve high $T_{c}$ if the $d_{x y}$ and $d_{x^{2}-y^{2}}$ orbitals remain close to degenerate, for example, in a hexagonal lattice structure where an anion is shared by only two NN cation atoms. We will discuss this case elsewhere. This discussion suggests that there are many lattice-structure variations for proposed new high- $T_{c}$ superconductors because of the flexibility of the trigonal bipyramidal complex.

We can estimate the possible highest $T_{c}$ that could be achieved in these systems. As a rough estimation, we can compare the energy scales of the effective models with those of cuprates and iron-based superconductors. In cuprates, the NN effective hopping parameter induced through the $d-p$ hybridization is about $0.43 \mathrm{eV}$ [11]. In iron-based superconductors, it is the next NN (NNN) effective hopping parameters induced primarily by the $d-p$ hybridization. The values of the NNN hopping parameters range from 0.15 to $0.25 \mathrm{eV}$ [12], depending on materials and orbitals. Thus, the energy scale in ironbased superconductors is roughly half of the energy scale in cuprates. The highest $T_{c}$ in iron-based superconductors is also around the half of the value achieved in cuprates. In the fitted TB model in Table I for $\mathrm{YNiO}_{3}$, the NN hopping is about $0.31 \mathrm{eV}$. Therefore, we expect that the highest $T_{c}$ here is at least comparable to those in iron-based superconductors. Namely, it should be over $50 \mathrm{k}$. It can also be estimated by the AFM exchange coupling strength. In cuprates, the NN AFM coupling is about $0.12 \mathrm{eV}$ [11], and in iron-based superconductors, the magnetic exchange coupling is about $0.05 \mathrm{eV}$ [13]. As shown in the Appendix, the magnetic exchange coupling in $\mathrm{YNiO}_{3}$ is estimated to be $0.1 \mathrm{eV}$. Thus, as $T_{c}$ is expected to be scaled with the AFM exchange coupling, it is reasonable to expect that $T_{c}$ in the predicted electronic environment should be between those of cuprates and iron-based superconductors. It is important to note that the above estimation is only for the possible maximum $T_{c}$. The superconducting transition temperature in a superconductor, in general, is very sensitive to the detailed electronic structures, doping concentration, material quality, possible competing orders, and many other factors. For example, $T_{c}$ for a $d+i d$ pairing state can be very sensitive to impurities so that a high-quality crystal may need to be obtained to maximize $T_{c}[14,15]$.

It is interesting to compare the proposed electronic structure with those of the layered sodium cobalt oxyhydrate $\mathrm{NaCoO}_{2}$, which owns a trigonal cobalt oxygen lattice [16]. However, the triangular cobalt lattice is built by edge-shared $\mathrm{CoO}_{6}$ octahedral complexes. The $\mathrm{NN}$ hopping between two Co atoms stems from the $d$ - $d$ direct chemical bonds. Thus, even if the strong electron-electron correlation has been argued in this material [17], the material violates our basic principles so that it is not a candidate for high- $T_{c}$ superconductivity. In other words, the true superexchange couplings in $\mathrm{NaCoO}_{2}$ have a much lower energy scale than those in the predicted electronic structures of this paper. This may be the primary reason why only low $T_{c}$ was achieved in doped $\mathrm{NaCoO}_{2}$ compounds.

We can also design the similar structure with $4 d$ or $5 d$ transition-metal elements as cation atoms in the $4 d^{7}$ or $5 d^{7}$ filling configuration. In the Appendix, we provide the band structure of Pd-based materials in which $\mathrm{Pd}^{3+}$ is in a $4 d^{7}$ filling configuration. The essential $\alpha$ band is very similar to the above results. Although the correlation effect is generally weakened in heavier transition-metal systems, the robust $\alpha$ band suggests that the proposed class of high- $T_{c}$ superconductors may include many series of materials.

While there is no material in current material databases that hosts the electronic environment described in this paper, it is encouraging to notice that $\mathrm{CoO}_{5}$ trigonal bipyramidal complexes have been appeared in recently synthesized material $\mathrm{LiBCoO}_{3}[18]$ in which $\mathrm{Co}$ is $\mathrm{Co}^{2+}$ with a $d^{7}$ filling configuration.

In summary, we predict that high- $T_{c}$ superconductivity exists in a triangle lattice formed by the cation-anion trigonal bipyramidal complexes close to a $d^{7}$ filling configuration on the cation ions. The predicted Co- and Ni-based superconductors or corresponding $4 d$ and $5 d$ transition-metal-based superconductors should have a robust $d_{x y} \pm i d_{x^{2}-y^{2}}$ pairing symmetry. If the prediction is verified, together with cuprates and iron-based superconductors, it can convincingly establish the high- $T_{c}$ superconducting mechanism and also pave the way to

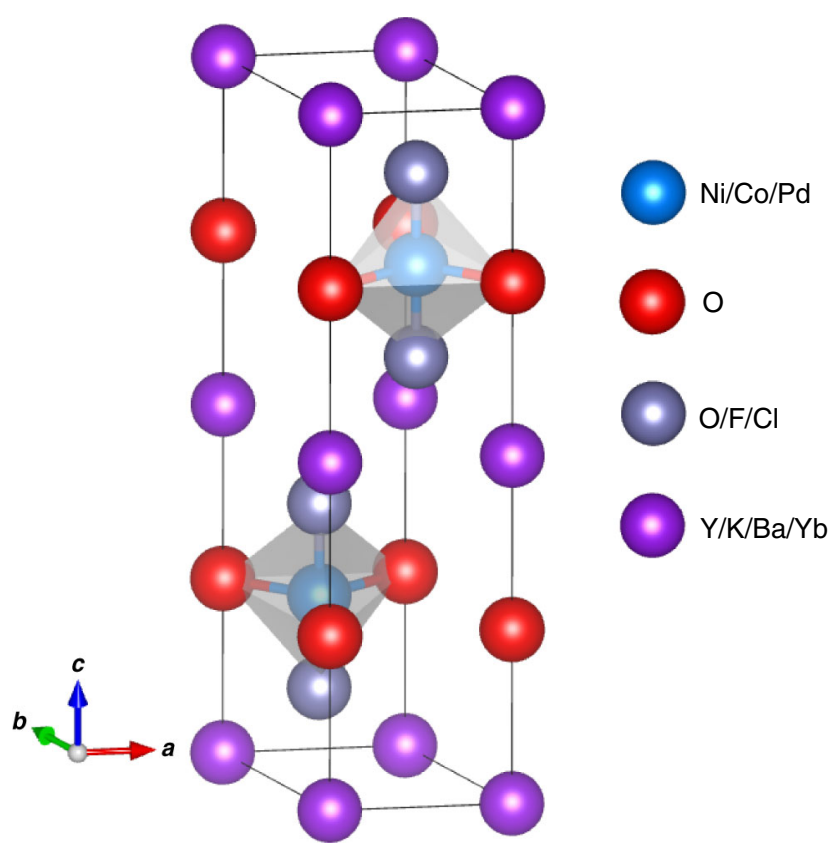

FIG. 5. The crystal structure of $A M O X_{2}(A=\mathrm{Y}, \mathrm{K}, \mathrm{Ba}, \mathrm{Yb}$; $M=\mathrm{Ni}, \mathrm{Co}, \mathrm{Pd}$; and $X=\mathrm{Cl}, \mathrm{O})$. 
design and search for new unconventional high- $T_{c}$ superconductors.

We thank D. L. Feng for useful discussion. The work is supported by the National Basic Research Program of China, National Natural Science Foundation of China (NSFC), and the Strategic Priority Research Program of Chinese Academy of Sciences.

\section{APPENDIX: RESULTS FROM FIRST-PRINCIPLES CALCULATIONS}

In this Appendix, we investigate the electronic structures of possible materials constructed by the trigonal bipyramidal complexes under $3 d^{7}$ filling configurations. Our density-functional-theory calculations employ the projector- augmented-wave method encoded in the
Vienna ab initio simulation package (VASP) [19-21], and the GGA for the exchange-correlation functional is used [22]. The cutoff energy of $500 \mathrm{eV}$ is taken for expanding the wave functions into plane-wave basis. In the calculation, the number of these $k$ points is $(16 \times 16 \times 6)$ for the nonmagnetic calculations and $(9 \times 9 \times 5)$ for the magnetic calculations. The GGA plus on-site repulsion $U$ method $(\mathrm{GGA}+U)$ in the formulation of Dudarev et al. [23] is employed to describe the electron-correlation effect associated with the $\mathrm{Ni}$ and Co $3 d$ states by an effective parameter $U_{\text {eff }}$. The value of $U_{\text {eff }}=4 \mathrm{eV}$ on $\mathrm{Ni}$ and Co is adopted in the calculations. The lattice constants and internal atomic positions are fully relaxed.

Figure 5 shows the crystal structure of $\operatorname{AMOX}_{2}(A=\mathrm{Y}$, $\mathrm{K}, \mathrm{Ba}, \mathrm{Yb} ; M=\mathrm{Ni}, \mathrm{Co}, \mathrm{Pd}$; and $X=\mathrm{F}, \mathrm{Cl}, \mathrm{O})$. The transition-metal ions are surrounded by trigonal
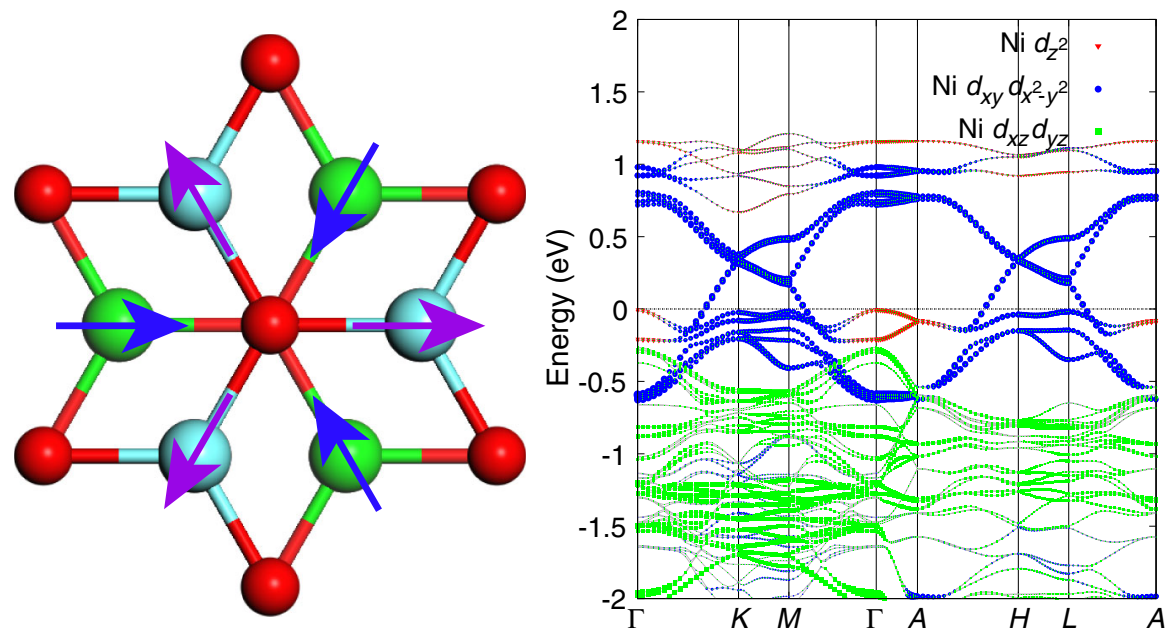

FIG. 6. The in-out AFM state and the GGA $+U$ band structure of this state for $\mathrm{YNiO}_{3}$.
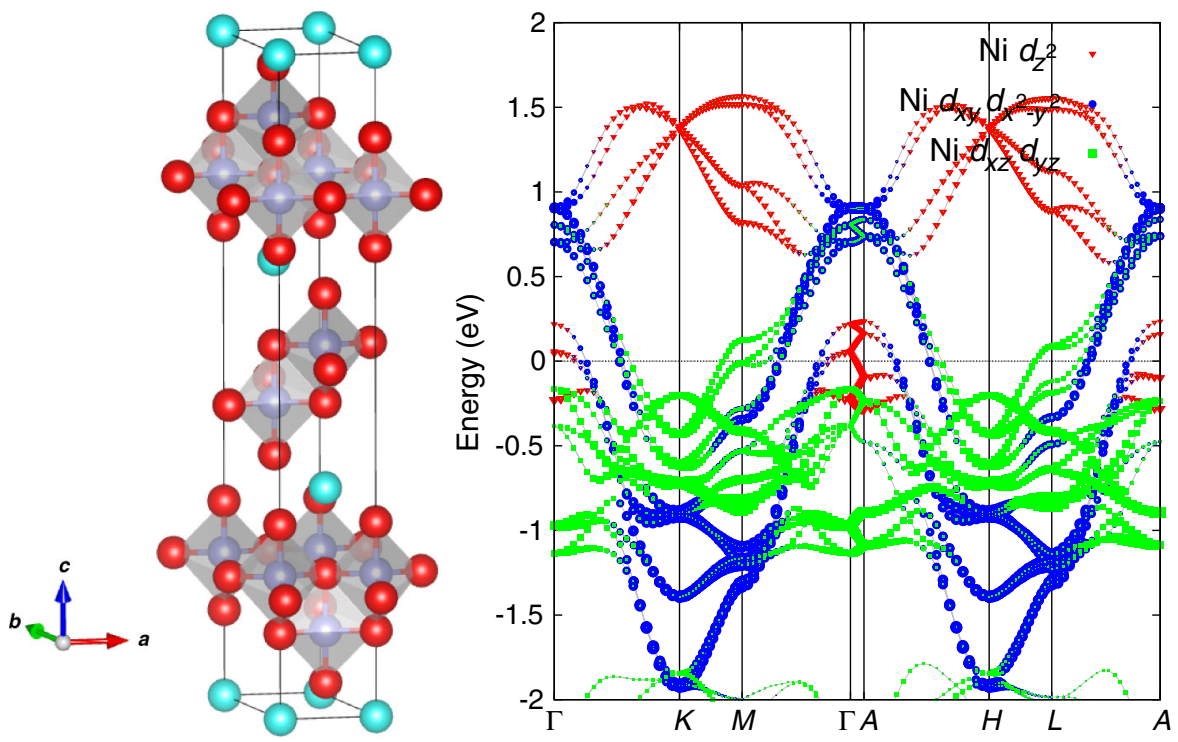

FIG. 7. The crystal structure and band structure of $\mathrm{YbNi}_{2} \mathrm{O}_{4}$. 
bipyramidal arrangements of anions. The band structure of $\mathrm{YNiO}_{3}$ has been given in the main text. The Fermi surfaces are mainly contributed by $d_{x y}$ and $d_{x^{2}-y^{2}}$ orbitals of Ni. To investigate the magnetic properties, we perform the GGA $+U$ calculations for different magnetic states. The in-out AFM magnetic state in the triangle lattice is found to have a large energy gain about $0.35 \mathrm{eV}$ per Ni relative to the paramagnetic state. From this energy, we estimate that the nearest-neighbor AFM exchange couplings are about $0.1 \mathrm{eV}$, which is close to the values in cuprates. The band structure of $\mathrm{YNiO}_{3}$ in the in-out AFM state with GGA $+U$ $(U=4 \mathrm{eV})$ is shown in Fig. 6 .

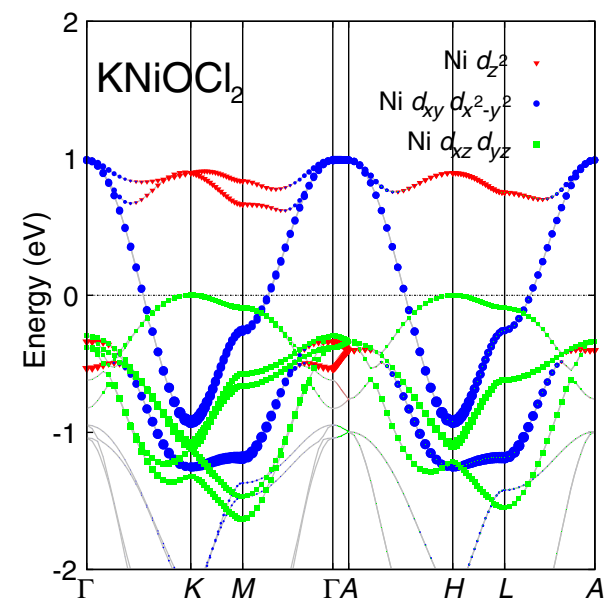

(a)

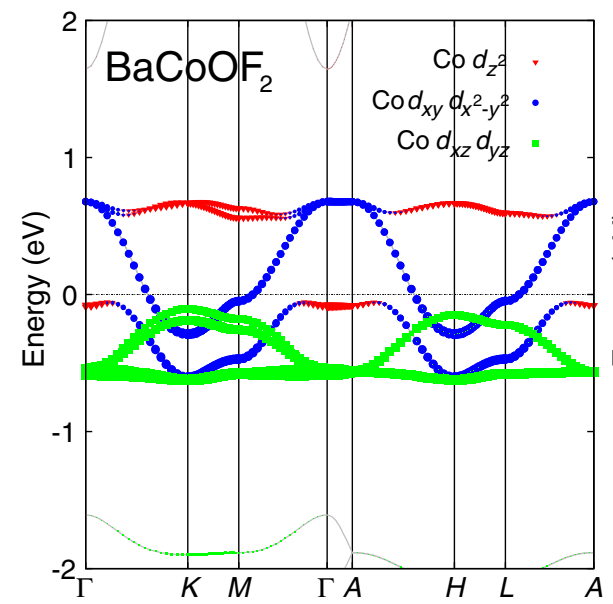

(c)

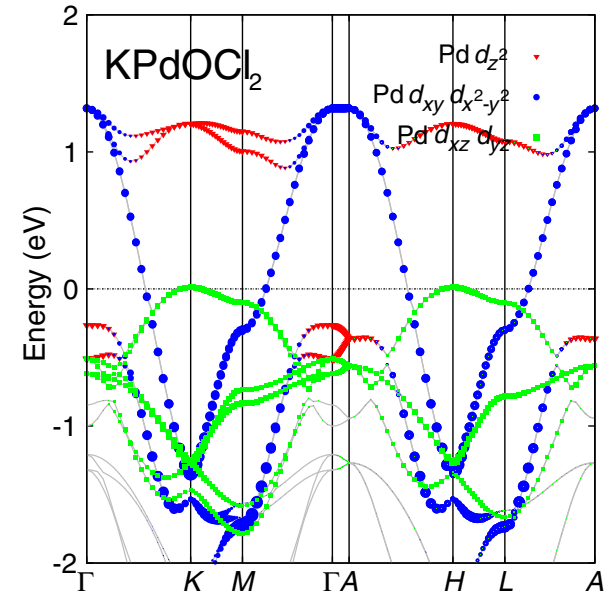

(e)

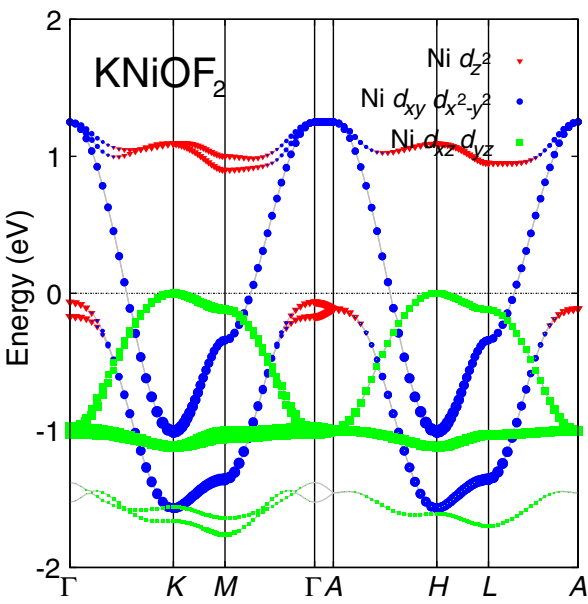

(b)

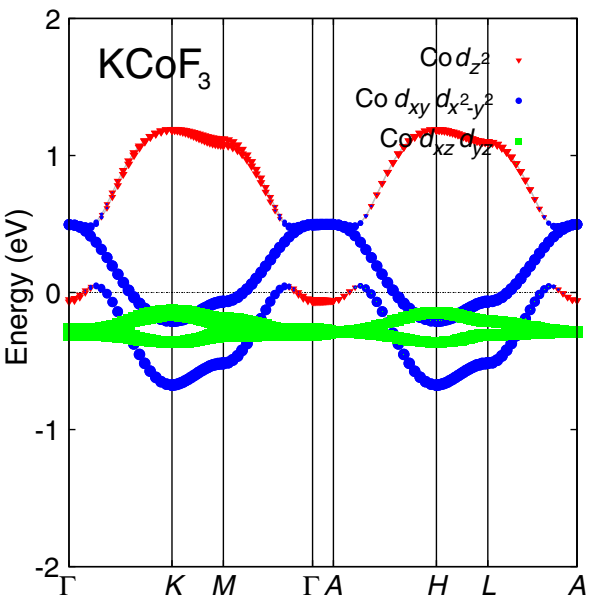

(d)

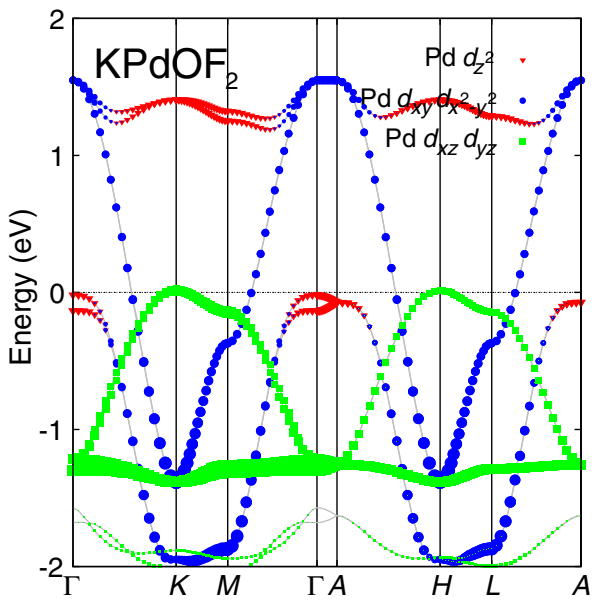

(f)

FIG. 8. The band structures of (a) $\mathrm{KNiOCl}_{2}$, (b) $\mathrm{KNiOF}_{2}$, (c) $\mathrm{BaCoOF}_{2}$, (d) $\mathrm{KCoF}_{3}$, (e) $\mathrm{KPdOCl}_{2}$, and (f) $\mathrm{KPdOF}_{2}$. 
Cuprates have many structural variations due to the presence of apical oxygens in the octahedral complex. Here, we can also consider a possible two-layer $\mathrm{NiO}$ material $\mathrm{YbNi}_{2} \mathrm{O}_{4}$, where the trigonal bipyramids in the two layers are connected by sharing edges. The crystal structure and band structure are given in Fig. 7. In contrast to $\mathrm{YNiO}_{3}, d_{y z}$ and $d_{x z}$ also contribute to the Fermi surfaces in $\mathrm{YbNi}_{2} \mathrm{O}_{4}$ due to the strong coupling between the two $\mathrm{NiO}$ layers. This result suggests that this multilayer structure likely will not enhance $T_{c}$, in contrast to cuprates.

We also consider other possible Ni-, Co-, and Pd-based materials with the trigonal bipyramidal crystal field, whose band structures are shown in Fig. 8. We find that the states near the Fermi level are mainly attributed to $d_{x y}$ and $d_{x^{2}-y^{2}}$ orbitals in all materials. For the Ni-based materials, the effective hopping though the anions between the nearestneighbor $\mathrm{Ni}$ is large, making the $d_{x y}$ and $d_{x^{2}-y^{2}}$ bands dispersive. For the Pd-based materials, the $d_{x y}$ and $d_{x^{2}-y^{2}}$ bandwidth is larger due to the extended $4 d$ orbitals. However, the effective hopping in Co-based materials is much smaller. These results suggest that there are rich classes of materials to explore the correlated electron physics.

In summary, there are many possible materials that can provide the desired electronic structure proposed in the main text. One can also replace elements in these materials by other elements with an identical valance to search stable crystal structures. High-temperature superconductivity should be induced by doping these materials.

[1] J.G. Bednorz and K. A. Muller, Possible High $T_{c}$ Superconductivity in the Ba-La-Cu-o System, Z. Phys. B 64, 189 (1986).

[2] Y. Kamihara, T. Watanabe, M. Hirano, and H. Hosono, Iron-Based Layered Superconductor $\mathrm{La}\left[\mathrm{O}_{1-x} \mathrm{~F}_{x}\right] \mathrm{FeAs}$ $(x=0.05-0.12)$ with $T_{c}=26 \mathrm{~K}$, J. Am. Chem. Soc. 130, 3296 (2008).

[3] D. C. Johnston, The Puzzle of High Temperature Superconductivity in Layered Iron Pnictides and Chalcogenides, Adv. Phys. 59, 803 (2010).

[4] J. Hu, Robustness of s-Wave Pairing Symmetry in Iron-Based Superconductors and Its Implications to Fundamentals on Magnetically-Driven High Temperature Superconductivity, arXiv:1506.05791.

[5] J. Hu and H. Ding, Local Antiferromagnetic Exchange and Collaborative Fermi Surface as Key Ingredients of High Temperature Superconductors, Sci. Rep. 2, 381 (2012).

[6] J. S. Davis and D.-H. Lee, Concepts Relating Magnetic Interactions, Intertwined Electronic Orders, and Strongly Correlated Superconductivity, Proc. Natl. Acad. Sci. U.S.A. 110, 17623 (2013).
[7] H. L. Yakel, W. C. Koehler, E. F. Bertaut, and E. F. Forrat, On the Crystal Structure of the Manganese(III) Trioxides of the Heavy Lanthanides and Yttrium, Acta Crystallogr. 16, 957 (1963).

[8] G. A. Smolenskii and V. A. Bokov, Coexistence of Magnetic and Electric Ordering in Crystals, J. Appl. Phys. 35, 915 (1964).

[9] K. Manabu, S. Miura, Y. Nakagawa, N. Kimizuka, I. Shindo, and K. Siratori, Magnetization of $\mathrm{YbFe}_{2} \mathrm{O}_{4}+x$, J. Phys. Soc. Jpn. 51, 2801 (1982).

[10] M. K. Wu, J. R. Ashburn, C. J. Torng, P. H. Hor, R. L. Meng, L. Gao, Z. J. Huang, Y. Q. Wang, and C. W. Chu, Superconductivity at $93 \mathrm{~K}$ in a New Mixed-Phase $\mathrm{Y}$-Ba-Cu-O Compound System at Ambient Pressure, Phys. Rev. Lett. 58, 908 (1987).

[11] M. R. Norman and C. Pepin, The Electronic Nature of High Temperature Cuprate Superconductors, Rep. Prog. Phys. 66, 1547 (2003).

[12] K. Kuroki, S. Onari, R. Arita, H. Usui, Y. Tanaka,, H. Kontani, and H. Aoki, Unconventional Pairing Originating from the Disconnected Fermi Surfaces of Superconducting $\mathrm{LaFeAsO}_{1-x} \mathrm{~F}_{x}$, Phys. Rev. Lett. 101, 087004 (2008).

[13] P. C. Dai, J. Hu, and E. Dagotto, Magnetism and Its Microscopic Origin in Iron-Based High-Temperature Superconductors, Nat. Phys. 8, 709 (2012).

[14] A. V. Balatsky, I. Vekhter, and J.-X. Zhu, Impurity-Induced States in Conventional and Unconventional Superconductors, Rev. Mod. Phys. 78, 373 (2006).

[15] R. Joynt and L. Taillefer, The superconducting phases of $\mathrm{UPt}_{3}$, Rev. Mod. Phys. 74, 235 (2002).

[16] K. Takada, H. Sakurai, E. Takayama-Muromachi, F. Izumi, R. A. Dilanian, and T. Sasaki, Superconductivity in Two-Dimensional $\mathrm{CoO}_{2}$ Layers, Nature (London) 422, 53 (2003).

[17] D. J. Singh, Electronic structure of $\mathrm{NaCo}_{2} \mathrm{O}_{4}$, Phys. Rev. B 61, 13397 (2000).

[18] S. H. Bo, G. M. Veith, M. R. Saccomanno, H. Huang, P. V. Burmistrova, A. C. Malingowski, R. L. Sacci, K. R. Kittilstved, C. P. Grey, and P. G. Khalifah, Thin-Film and Bulk Investigations of $\mathrm{LiCoBO}_{3}$ as a Li-Ion Battery Cathode, Appl. Mater. Int. 6, 10840 (2014).

[19] G. Kresse and J. Hafner, Ab Initio Molecular-Dynamics for Liquid-Metals, Phys. Rev. B 47, 558 (1993).

[20] G. Kresse and J. Furthmuller, Efficient Iterative Schemes for Ab Initio Total-Energy Calculations Using a Plane-Wave Basis Set, Phys. Rev. B 54, 11169 (1996).

[21] G. Kresse and J. Furthmuller, Efficiency of Ab-Initio Total Energy Calculations for Metals and Semiconductors Using a Plane-Wave Basis Set, Comput. Mater. Sci. 6, 15 (1996).

[22] J. P. Perdew, K. Burke, and M. Ernzerhof, Generalized Gradient Approximation Made Simple, Phys. Rev. Lett. 77, 3865 (1996).

[23] S. L. Dudarev, G. A. Botton, S. Y. Savrasov, C. J. Humphreys, and A.P. Sutton, Electron-Energy-Loss Spectra and the Structural Stability of Nickel Oxide: An LSDA + U Study, Phys. Rev. B 57, 1505 (1998). 